\title{
Uso de una cargadora frontal cat 966h en la remoción de escombros
}

\section{Use of a cat $966 \mathrm{~h}$ front loader in debris removal}

Ing. Adolfo Juan Peña Pinargote, MSc

Universidad Internacional del Ecuador, Ecuador

Autor para correspondencia: ajpena@internacional.edu.ec

Fecha de recepción: 30 de agosto de 2018 - Fecha de aceptación: 01 noviembre de 2018

\section{Resumen}

El modelo $966 \mathrm{H}$ permite obtener un rendimiento que se puede sentir en las aplicaciones más exigentes. Esta máquina ofrece una inigualable eficiencia y comodidad para el operador en una cabina de clase mundial. Los nuevos cucharones de la Serie de rendimiento reducen los tiempos de excavación y permiten obtener mayores factores de llenado y mejor retención de material para incrementar la productividad y reducir el consumo de combustible. El revolucionario sistema electrohidráulico $(\mathrm{EH})$ proporciona una operación con la punta de los dedos y de bajo esfuerzo de los controles de elevación, de inclinación de la herramienta auxiliar. La fiabilidad, durabilidad y versatilidad del modelo $966 \mathrm{H}$ se reflejan en una máquina con mejor construcción para satisfacer sus necesidades.

Palabras Clave: rendimiento; aplicaciones exigentes; eficiencia y comodidad; productividad; versatilidad; satisfacer necesidades

\begin{abstract}
The $966 \mathrm{H}$ model provides performance that can be felt in the most demanding applications. This machine offers unmatched efficiency and operator comfort in a world class cab. The new ladles Performance Series reduces dig times and allow for greater fill factors and better material retention to increase productivity and reduce fuel consumption. The revolutionary electrohydraulic (EH) provides an operation with the fingertips and low effort controls lifting, tilting of the auxiliary tool. Reliability, durability and versatility $966 \mathrm{H}$ model are reflected on a machine with better construction to meet your needs.
\end{abstract}

Key words: performance demanding applications; efficiency and comfort; productivity; versatility; meet needs 


\section{Introducción}

Habiendo sucedido los lamentables sucesos del terremoto del 16 de abril del 2016 que tuvo como resultado la caída de una vivienda en una calle en la Cdla El Limonal se pudo constatar que los escombros están afectando el tránsito vehicular por lo que se requiere de una cargadora frontal y analizando la necesidad tuvimos el resultado que la cargadora frontal CAT $966 \mathrm{H}$ es la que se adapta a nuestras necesidades ya que los componentes que utilizan los cargadores de ruedas Cat están diseñados y fabricados según los estándares de calidad Caterpillar para garantizar un máximo rendimiento, incluso en condiciones de operación extremas. (Revista Motor)

Debido a que los componentes de servicio pesado reducen el riesgo de desgaste prematuro, se aumenta la disponibilidad y se reducen los costos de operación durante la vida útil de la máquina.

La Serie $\mathrm{H}$ cuenta con muchos componentes que influyen en los diseños de productos que han producido máquinas fiables y duraderas durante generaciones. El modelo $966 \mathrm{H}$ está diseñado para complementar su plan comercial, reducir las emisiones y minimizar el consumo de recursos naturales. (Yépez, 2012)

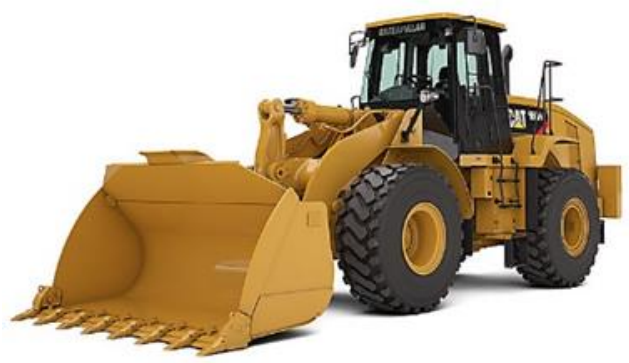

Figura 1. Cargadora frontal $966 \mathrm{H}$

(Cat, 2017)

Además, tiene un excelente rendimiento palpable con la capacidad para trabajar en las aplicaciones más exigentes. Comodidad y eficiencia sin igual del operador en una cabina de calidad mundial. Controles electrónicos e hidráulicos revolucionarios para una operación con el menor esfuerzo. Aumento de la productividad con menores costos de posesión y operación. (Yépez, 2012)

\section{Materiales y Métodos}

\section{Especificaciones técnicas}

Hay que tener en cuenta que las especificaciones técnicas están sujetas a cambios estos varían dependiendo del modelo y tipo de uso que se le dé al cargador frontal. (CatR, 2016)

Tabla 1. Especificaciones técnicas de la transmisión de la cargadora (CatR, 2016) 


\begin{tabular}{|l|l|}
\hline Transmisión & \multicolumn{1}{|c|}{ transmisión con cambio de fuerza de velocidades } \\
\hline " Tipo de transmisión & Número de marchas adelante \\
\hline " Número de marchas atrás & \\
\hline " Velocidad máxima hacia \\
adelante
\end{tabular}

Tabla 2. Especificaciones técnicas de frenos, ejes y neumáticos de la cargadora (Cat, 2017)

\begin{tabular}{|c|c|}
\hline \multicolumn{2}{|l|}{ Frenos } \\
\hline 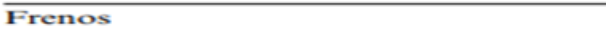 & $\begin{array}{l}\text { Cumple con las } \\
\text { normas requeridas }\end{array}$ \\
\hline \multicolumn{2}{|l|}{ - Cumple con los estándares ISO 3450-2011. } \\
\hline \multicolumn{2}{|l|}{ Ejes } \\
\hline Delantero & Parte delantera fija \\
\hline Trasero & Oscilante $\pm 13^{\circ}$ \\
\hline Subida y bajada máxima de una rueda & $502 \mathrm{~mm} \quad 19,8^{*}$ \\
\hline \multicolumn{2}{|l|}{ Neumáticos } \\
\hline Neumáticos & $\begin{array}{l}\text { Elija entre una amplia } \\
\text { variedad de neumáticos para } \\
\text { adaptarse a su aplicación. }\end{array}$ \\
\hline 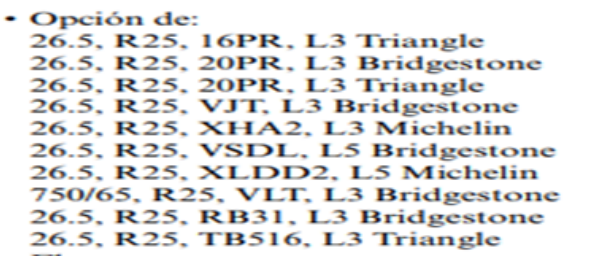 & \\
\hline
\end{tabular}

\section{Características}

\section{Estructura del chasis de la cargadora frontal Cat $966 \mathrm{H}$}

El diseño de bastidor articulado del $966 \mathrm{H}$ dispone de un bastidor de motor duradero de sección en caja y una torre de cargador de cuatro placas rígidas soldadas con un robot. La soldadura con robot crea articulaciones del bastidor con soldaduras de penetración profunda en las placas y una fusión excelente para lograr una resistencia y una durabilidad máximas.

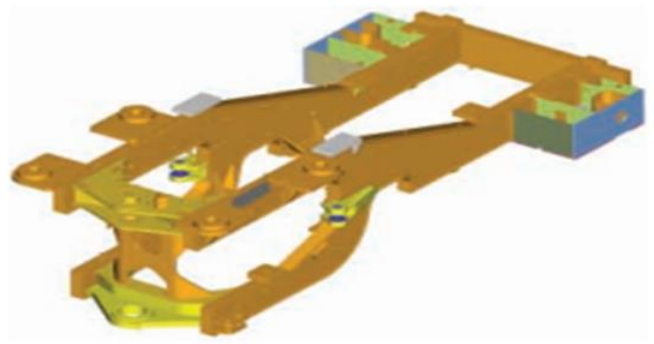


Figura 2. Estructura del chasis de la cargadora frontal Cat $966 \mathrm{H}$

(Cat, 2017)

Cuenta con un sistema de enganche extendido el cual la distancia entre las placas de enganche superior e inferior contribuye de forma importante al rendimiento de la máquina y a la duración de los componentes. El diseño de enganche extendido de Caterpillar proporciona una distribución de carga y una duración de los cojinetes excelentes. Tanto los pasadores de enganche superiores como inferiores pivotan sobre cojinetes de rodillos cónicos dobles aumentando la durabilidad al distribuir las cargas verticales y horizontales sobre una superficie mayor. La abertura ancha también permite un acceso de servicio excelente. (Tracsa, 2017)

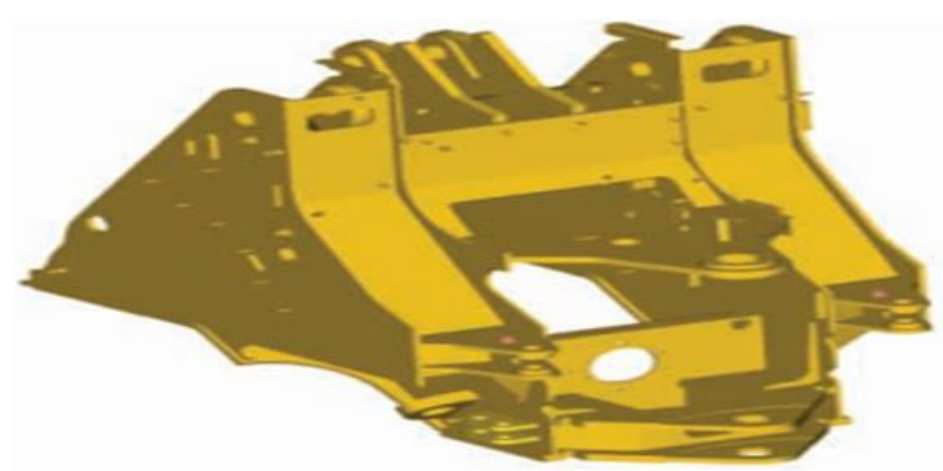

Figura 3. Sistema de enganche de la cargadora frontal Cat $966 \mathrm{H}$ (Cat, 2017)

El contrapeso de una pieza está integrado en el diseño y el estilo del $966 \mathrm{H}$. Este contrapeso de $1.142 \mathrm{~kg}(2.517 \mathrm{lb})$ incorpora las luces traseras en la parte superior de la estructura.

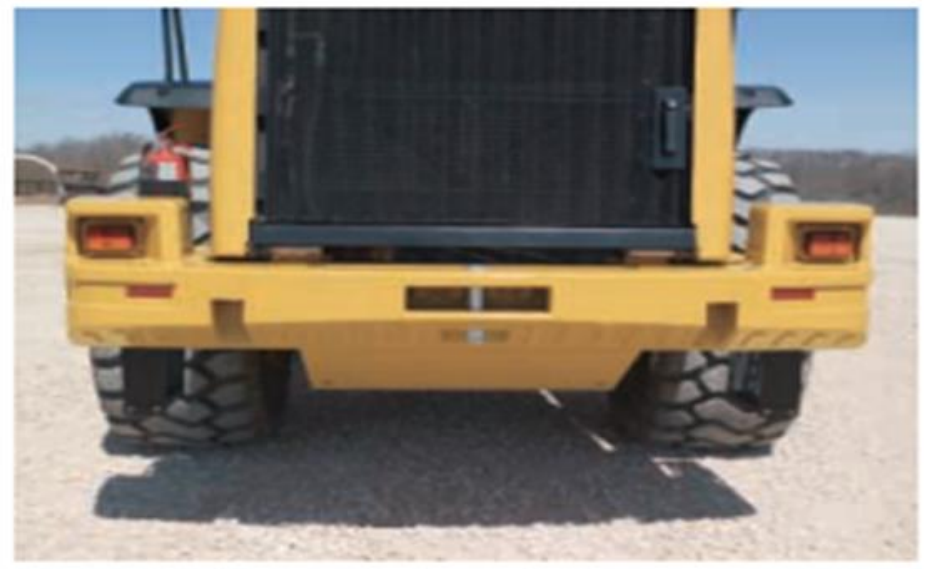

Figura 4. Sistema de enganche de la cargadora frontal Cat $966 \mathrm{H}$ (Cat, 2017)

El varillaje del $966 \mathrm{H}$ tiene un diseño de barra en $\mathrm{Z}$ de inclinación individual. El varillaje de barra en $\mathrm{Z}$ genera una fuerza de desprendimiento excelente y un buen ángulo de inclinación hacia atrás para poder cargar mejor el cucharón y retener la carga. Los brazos de levantamiento son de acero macizo y proporcionan una fuerza superior con un área de visión excelente del extremo delantero. Este diseño ofrece un alcance y un espacio libre de descarga excelentes que 
se adapta de forma excepcional a los camiones de obras y a los de transporte por carretera. Dispone de sensores giratorios para la palanca de inclinación y el circuito de levantamiento que permiten al operador fijar electrónicamente las posiciones de tope desde la cabina. Tiene un protector que cubre el sensor para protegerlo contra los daños. (Nicholas, 2010)

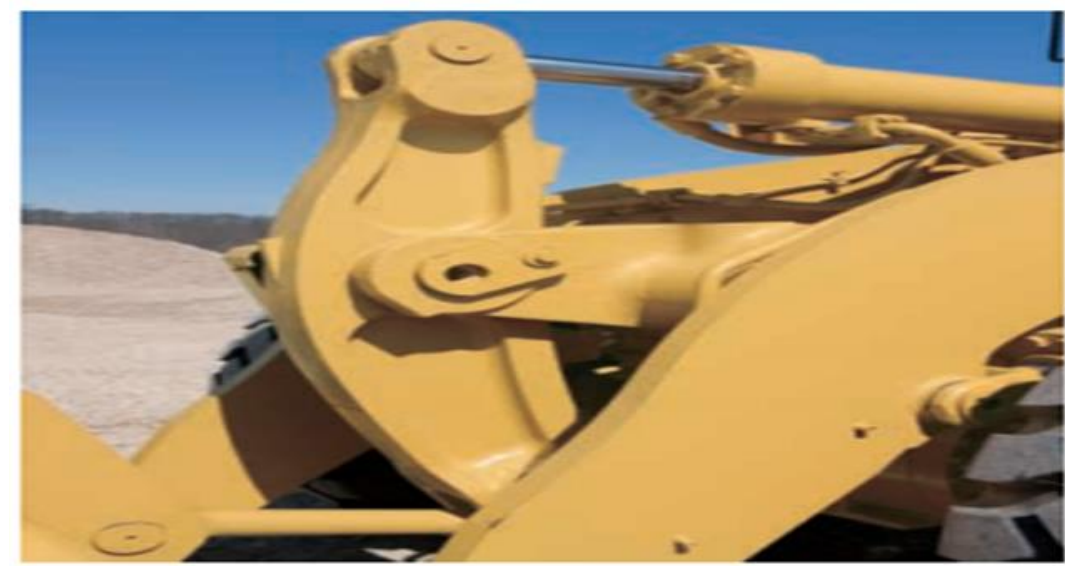

Figura 5. Inclinación de varillaje en $Z$ del cucharon de carga de la cargadora.

(Cat, 2017)

\section{Sistema Hidráulico de la cargadora Cat $966 \mathrm{H}$}

El 966H tiene un sistema hidráulico de detección de carga que se ajusta automáticamente a las condiciones de operación para suministrar solamente el caudal hidráulico necesario por el accesorio a fin de mejorar la eficiencia del combustible.

Los operadores observarán una mayor facilidad de operación, mayor tracción en las ruedas dentro de la pila y un aumento del $20 \%$ en la fuerza de levantamiento.

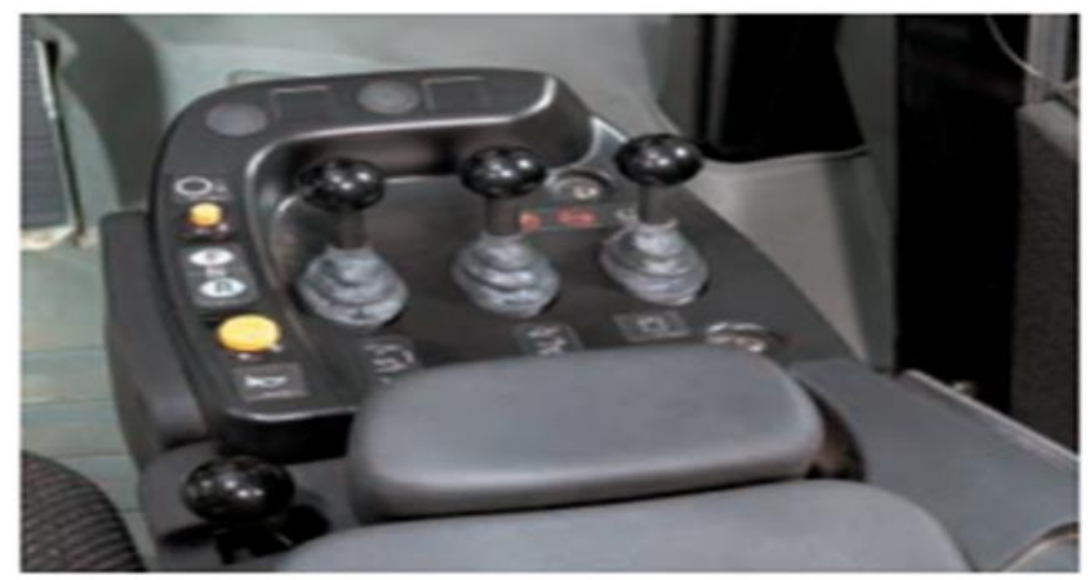

Figura 6. Sistemas de mandos hidráulicos de la cargadora Cat $966 \mathrm{H}$

(CatR, 2016)

\section{Sistema de enfriamiento de la cargadora $966 \mathrm{H}$}


Muchos cargadores de la competencia usan sistemas de enfriamiento que extraen aire de los lados, a través del compartimiento del motor y lo expulsa por la parte trasera de la máquina. El sistema de enfriamiento del $966 \mathrm{H}$ está aislado del compartimiento del motor por medio de un protector no metálico. El ventilador de velocidad variable accionado hidráulicamente extrae aire limpio de la parte trasera de la máquina y lo expulsa por los lados y la parte superior del capó. Los resultados finales son una eficiencia de enfriamiento óptima, mayor eficiencia de combustible, menos obstrucciones del radiador y niveles de sonido reducidos para el operador.

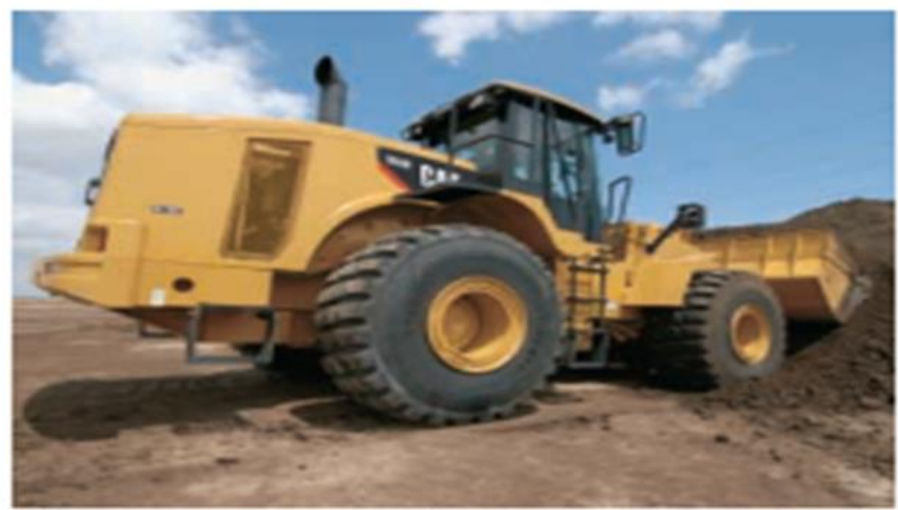

Figura 7. Ventilación lateral de la cargadora Cat $966 \mathrm{H}$.

(CatR, 2016)

\section{Resultados}

\section{Sistema de transmisión de la cargadora 966H}

Servo transmisión planetaria: El servo transmisión planetaria electrónica con capacidad de cambios automáticos está diseñada y fabricada por Caterpillar. La velocidad altamente sensible de potencia máxima y los cambios de dirección proporcionan unos ciclos y una productividad excelentes.

Control de cambios variable. Adapte las configuraciones de cambios de la transmisión a los requisitos de las aplicaciones de la máquina. El Control de Cambios Variable (VSC) mejora la calidad de los cambios y la eficiencia del combustible en ciertas aplicaciones dejando que la transmisión efectúe un cambio ascendente a menores rpm del motor.

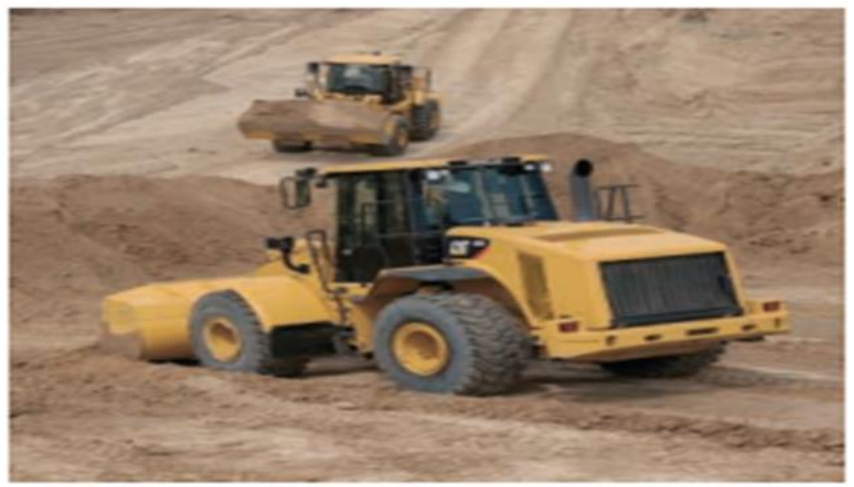

Figura 8. Sistema de transmisión planetaria de la cargadora Cat $966 \mathrm{H}$ 


\section{(CatR, 2016)}

\section{Sistema de Lubricación de la cargadora $966 \mathrm{H}$}

Cuenta con un sistema de lubricación automática optativo de Caterpillar proporciona una lubricación precisa y automática de los pasadores y bujes - durante la operación del cargador. La lubricación automática reduce el tiempo pasado en el mantenimiento diario y el tiempo de inactividad para reparaciones no planificadas debido a un engrase inadecuado - aumentando la productividad.

\section{Sistema de suspensión de la cargadora $966 \mathrm{H}$}

El sistema de control de amortiguación optativo mejora la amortiguación, el rendimiento y la retención de carga cuando se desplaza sobre terreno irregular. Los operadores toman confianza desplazándose a mayores velocidades en las operaciones de carga y transporte disminuyendo los tiempos de los ciclos y aumentando la productividad.

\section{Versatilidad de la cargadora $966 \mathrm{H}$}

Cuando tenga una operación especializada, necesitará un cargador de ruedas especializado. Para ser productivo. Se dispone de las siguientes configuraciones de máquinas para el $966 \mathrm{H}$ :

- Cargadores de agregados para patios.

- Aplicaciones forestales.

○ Cargador industrial.

- Cucharones de uso general.

- Cucharones para manipulación de materiales.

- Cucharones para roca.

- Cucharones para carbón.

- Cucharones de canteras para servicio pesado.

- Cucharones para rellenos sanitarios.

- Cucharón para limpieza y virutas de madera.

- Cucharones de uso múltiple.

- Cucharones de descarga lateral.

- Herramientas de corte.

- Brazos de manipulación de materiales.

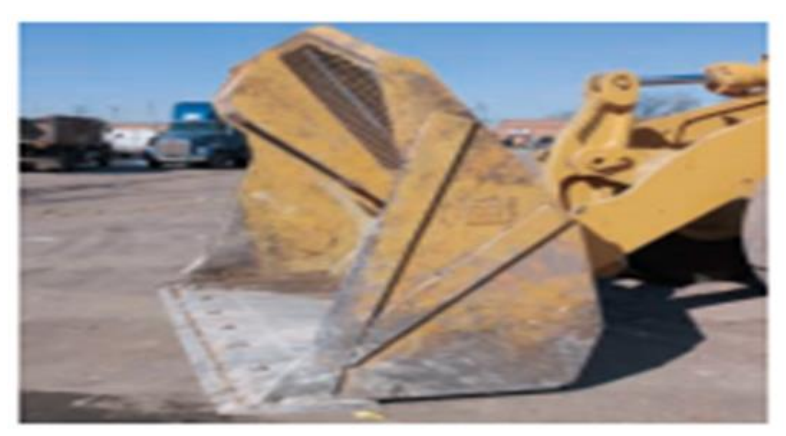


Figura 9. Pala cargadora de la cargadora Cat $966 \mathrm{H}$

(CatR, 2016)

\section{Cabina Del Conductor}

El 966H mantiene la distinción de ofrecer la cabina más grande y ergonómica de su clase.

Visibilidad: El $966 \mathrm{H}$ ofrece una visibilidad excelente de las partes delantera y trasera de la máquina. Tiene ventanas planas libres de distorsión que van hasta el piso de la cabina lo que permite una excelente visibilidad del cucharón. Los limpiaparabrisas de brazo húmedo en la parte delantera y trasera mantienen las ventanas limpias en cualquier condición.

El techo de la cabina: Tiene canales que guían el agua de lluvia hacia las esquinas de la cabina manteniendo limpias las ventanas. Posee unas proyecciones por todos los lados que protegen al operador contra el reflejo del sol.

Vibraciones: Caterpillar entiende que los cargadores de ruedas trabajan en algunos de los entornos más rigurosos. Al controlar las vibraciones normales de la máquina, se mejoran la eficiencia y la productividad del operador. El 966H Cat está diseñado de arriba a abajo con muchas características, tanto normales como optativas, que reducen las vibraciones.

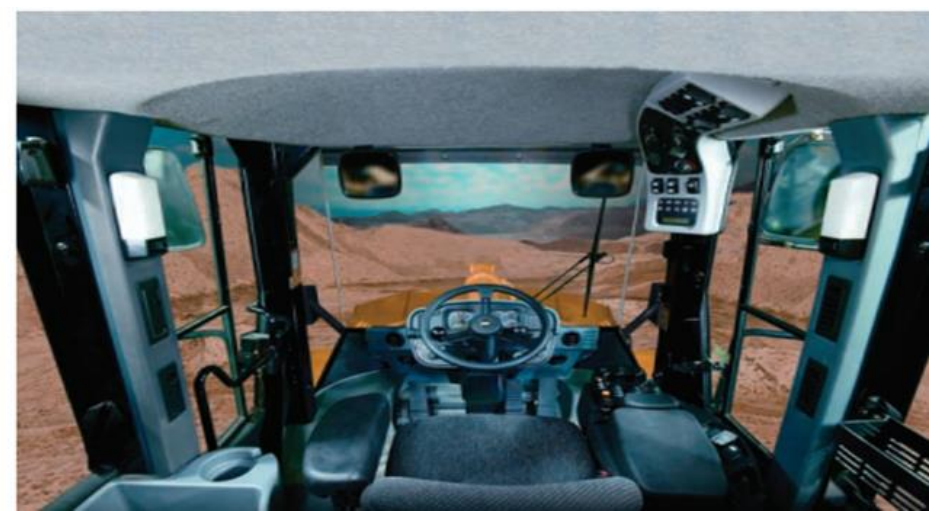

Figura 10. Cabina del conductor de la cargadora Cat $966 \mathrm{H}$.

Entrada y salida. Escalera con escalones de auto limpieza que mantiene la acumulación de escombros al mínimo. La escalera está inclinada $5^{\circ}$ hacia adelante para facilitar la entrada y la salida.

\section{Dirección}

El 966H ofrece una opción de sistemas de dirección para dar flexibilidad a su aplicación. Dirección convencional. La configuración de dirección convencional ofrece un sistema de dirección hidráulico con unidad de dosificación manual que requiere poco esfuerzo. La dirección con detección de carga transmite potencia al sistema de dirección sólo cuando se necesite. 
Cuando no se utilice el sistema de dirección, se dispone de más potencia del motor para generar fuerza de arrastre en las ruedas, fuerza de desprendimiento y fuerza de levantamiento, y resulta en un menor consumo de combustible. La columna de la dirección se inclina para máxima comodidad del operador.

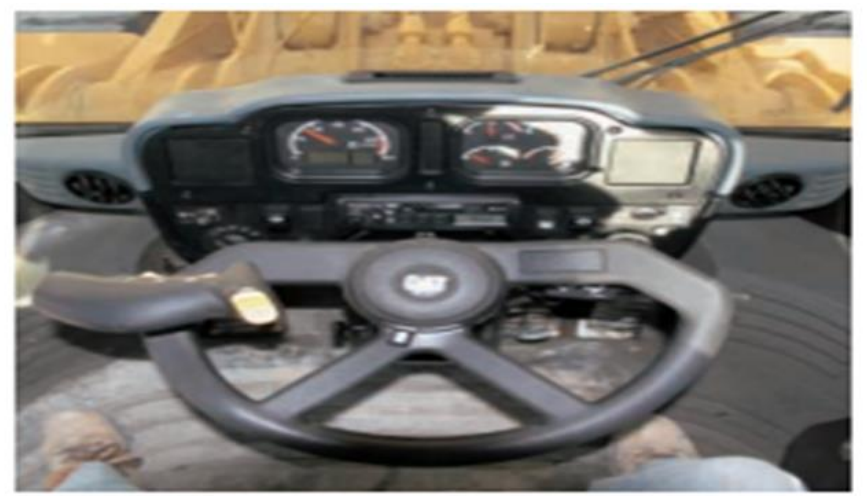

Figura 11. Cabina del conductor de la cargadora Cat $966 \mathrm{H}$.

\section{Características técnicas del motor de la cargadora Cat $966 \mathrm{H}$}

\begin{tabular}{|c|c|c|}
\hline \multirow{2}{*}{$\begin{array}{l}\text { Modelo de motor } \\
\text { Potencia bruta - SAE J1995 }\end{array}$} & \multicolumn{2}{|c|}{ C11 ACERT TM Cat $^{*}$} \\
\hline & $213 \mathrm{~kW}$ & $286 \mathrm{hp}$ \\
\hline Potencia neta - ISO 9249 & $195 \mathrm{~kW}$ & $262 \mathrm{hp}$ \\
\hline Potencia neta - SAE J1349 & $193 \mathrm{~kW}$ & $259 \mathrm{hp}$ \\
\hline Potencia neta - 80/1269/EEC & $195 \mathrm{~kW}$ & $262 \mathrm{hp}$ \\
\hline Par máximo (neto) a $1.400 \mathrm{rpm}$ & $1215 \mathrm{~N}-\mathrm{m}$ & 896 lb-pie \\
\hline Calibre & $130 \mathrm{~mm}$ & 5,12 pulg \\
\hline Carrera & $140 \mathrm{~mm}$ & 5,51 pulg \\
\hline Cilindrada & $11.1 \mathrm{~L}$ & 677 pulg $^{2}$ \\
\hline \multicolumn{3}{|c|}{$\begin{array}{l}\text { Motor Caterpillar con tecnología ACERTTM - Cumple con } \\
\text { EPA Tier III, EU Stage III. }\end{array}$} \\
\hline \multicolumn{3}{|c|}{$\begin{array}{l}\text { - Estas clasificaciones se aplican a } 1.800 \text { rpm cuando la prueba } \\
\text { se realiza en las condiciones normales especificadas. }\end{array}$} \\
\hline \multicolumn{3}{|c|}{$\begin{array}{l}\text { - Clasificación para la potencia neta anunciada basada en la } \\
\text { potencia disponible cuando el motor esté equipado con alternador, } \\
\text { filtro de aire, silenciador y mando de ventilador hidráulico a petición } \\
\text { a la velocidad máxima del ventilador. }\end{array}$} \\
\hline
\end{tabular}

Figura 12. Cabina del conductor de la cargadora Cat $966 \mathrm{H}$.

\section{Sistema de frenos de la cargadora Cat $966 \mathrm{H}$}

El sistema de frenos de la cargadora cumple con las normas de OSHA, SAE J1473

OCT90 e ISO 3450-1985, por su versatilidad y confiabilidad al momento de ser utilizada sobre todo en maquinaria pesada.

\section{Neumáticos de la cargadora frontal Cat $966 \mathrm{H}$}

Los neumáticos de la cargadora se pueden elegir entre los siguientes:

- 26.5R25, L-2.

- $26.5 \mathrm{R} 25, \mathrm{~L}-3$ (XHA).

- 26.5R25, L-5 (VSDL).

- 26.5-25, L-3 (SRG, SHRL). 
- $750 / 65 \mathrm{R} 25, \mathrm{~L}-3$.

\section{Sistemas monitores de la cargadora Cat $966 \mathrm{H}$}

La supervisión del estado del producto simplifica la planificación de mantenimiento y reduce los costos, por lo que el Sistema Monitor Caterpillar (CMS) hace el seguimiento de los sistemas críticos de la máquina para alertar al operador de la necesidad potencial de servicio. Tres niveles de advertencia permiten al operador evaluar la situación con mayor precisión.

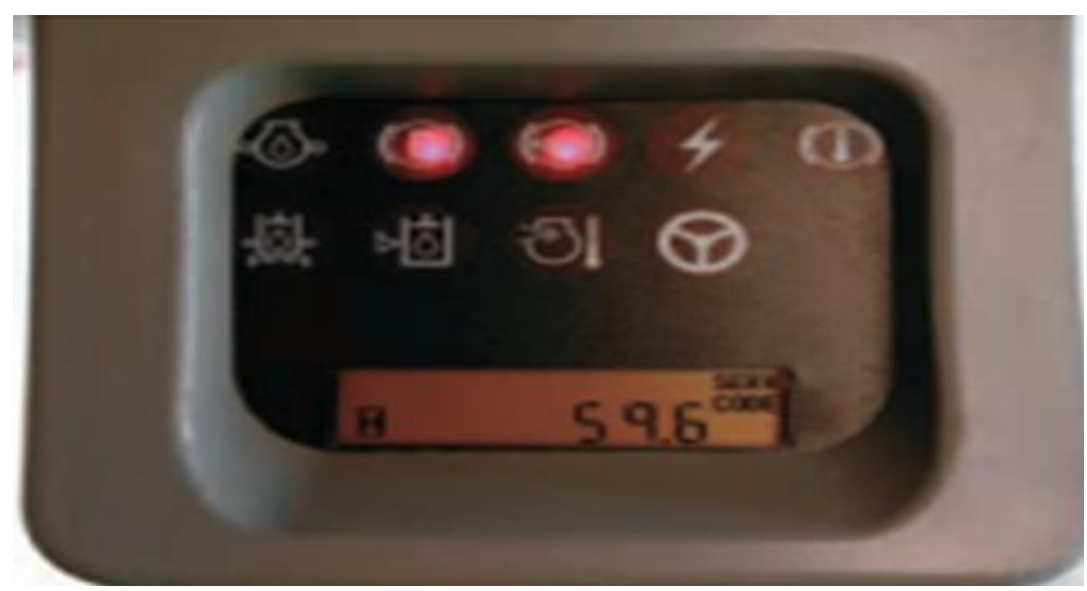

Figura 13. Sistema de monitoreo de la cargadora Cat $966 \mathrm{H}$

\section{Capacidad de fluidos de la cargadora Cat $966 \mathrm{H}$}

Tabla 3. Capacidad de fluidos de la cargadora Cat $966 \mathrm{H}$

\section{Capacidades de llenado}

\begin{tabular}{lll}
\hline Tanque de combustible - Estándar & $380 \mathrm{~L}$ & $100,4 \mathrm{gal}$ \\
\hline Sistema de enfriamiento & $39 \mathrm{~L}$ & $10,3 \mathrm{gal}$ \\
\hline Cárter & $35 \mathrm{~L}$ & $9,25 \mathrm{gal}$ \\
\hline Transmisión & $44 \mathrm{~L}$ & $11,62 \mathrm{gal}$ \\
\hline $\begin{array}{l}\text { Diferenciales y mandos finales - } \\
\text { delanteros }\end{array}$ & $64 \mathrm{~L}$ & $16,9 \mathrm{gal}$ \\
$\begin{array}{l}\text { Diferenciales y mandos finales - } \\
\text { traseros }\end{array}$ & $64 \mathrm{~L}$ & $16,9 \mathrm{gal}$ \\
\hline Tanque hidráulico & $110 \mathrm{~L}$ & $29 \mathrm{gal}$
\end{tabular}

\section{Dimensiones de la cargadora Cat $966 \mathrm{H}$}




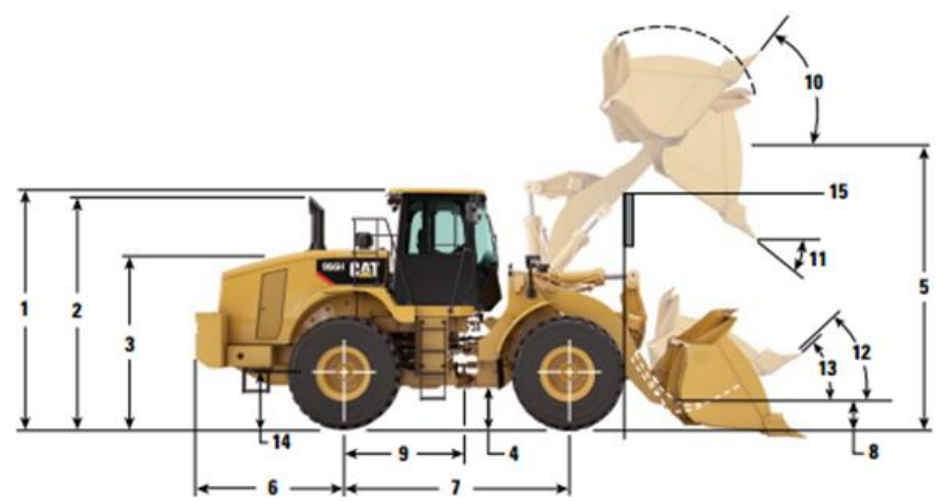

Figura 14. Dimensiones de la cargadora Cat $966 \mathrm{H}$

Tabla 4. Especificación de dimensiones de la cargadora Cat

\begin{tabular}{|c|c|c|}
\hline 1 Altura hasta la parte superior de ROPSIFOPS & $3.575 \mathrm{~mm}$ & $11 \cdot 7$ \\
\hline 2 Atura hasta la parte superior del tubo de escape & $3.524 \mathrm{~mm}$ & $11^{\prime} 6^{\circ}$ \\
\hline 3 Atura hasta la parte superior del capó & $2.655 \mathrm{~mm}$ & 87 \\
\hline 4 Espacio libre sobre el suelo con neumíticos Michelin 26.5 R25L.3 & $434 \mathrm{~mm}$ & $14^{\circ}$ \\
\hline 5 Altura del pasodor en B: estaindar & $4.235 \mathrm{~mm}$ & $1310^{\circ}$ \\
\hline Altura del pasador en B: kvantamixnto alto & $4.794 \mathrm{~mm}$ & $158^{\circ}$ \\
\hline 6 Desde la linea de centro del eje trasero hasta el eatremo del contrapeso & $2.279 \mathrm{~mm}$ & $75^{\circ}$ \\
\hline 1 Distancia entre ejes & $3.450 \mathrm{~mm}$ & $11^{\prime} 3^{*}$ \\
\hline 8 Atura del pasador en B en transporte: estindar & $641 \mathrm{~mm}$ & $21^{\circ}$ \\
\hline Atura del pasedor en B en transporte: kvantamiento alto & $788 \mathrm{~mm}$ & $26^{\circ}$ \\
\hline 9 Desde la linea central del eje trasero hasta el enganche & $1.725 \mathrm{~mm}$ & 57 \\
\hline 10 Inclinación hacia atrás en levantamiento míximo: estándar & \multicolumn{2}{|c|}{61 grados } \\
\hline Inclinación hacia atrás en levantamiento míximo: levantamiento alto & \multicolumn{2}{|c|}{71 grados } \\
\hline 11 Angulo de descarga a levantamiento máximo & \multicolumn{2}{|c|}{48,2 grados } \\
\hline 12 Inclinación hacia atrás en transporte: estándar & \multicolumn{2}{|c|}{50 grados } \\
\hline Inclinación hacia atrás en transporte: levantamiento alto & \multicolumn{2}{|c|}{49 grados } \\
\hline 13 Inclinación hacia atrás en tierra: estándar & \multicolumn{2}{|c|}{42 grados } \\
\hline Inclinación hacia atrás en tierra: kevantamiento alto & \multicolumn{2}{|c|}{39 grados } \\
\hline 14 Altura hasta la linea central del cje & $799 \mathrm{~mm}$ & 27 \\
\hline 15 Espacio libre del brazo de levantamiento a levantamiento estándar & $3.925 \mathrm{~mm}$ & $129^{\circ}$ \\
\hline Espacio libre del brazo de levantamiento a levantamien & $4,484 \mathrm{~mm}$ & 147 \\
\hline
\end{tabular}

\section{Conclusiones}

Como conclusiones que según las investigaciones que se realizó se pudo conocer el funcionamiento de este equipo pesado y su correcto uso en los diferentes trabajos que se pueden realizar con él, así mismo se pudo obtener los costos de los equipos así mismo como el alquiler de una máquina de estas las cuales sirven de gran ayuda.

\section{Bibliografía}

Admi. Corporation. (10 De 06 De 2017). Coches Sin Conductor. Obtenido De Http://Www.Cochesinconductor.Com/2017/06/Que-Desventajas-Tendran-Los-CochesAutonomos/

Beer, Johnston, Mazurek, \& Eisenberg. (2010). Mecánica Vectorial Para Ingenieros. Dinámica. México D.F: Mc Graw Hill. 
Casto Recio, David Barbado, Alejandro López \& Francisco Vera. (2014). Test De Campo Para Valorar La Resistencia De Los Músculos Del Tronco. Obtenido De Http://Www.Raco.Cat/Index.Php/Apuntsefd/Article/Viewfile/280885/368549

Cat. $(14 \quad$ De Marzo $\quad$ De $\quad$ 2017). Http://Www.Conexionescat.Com/Inicio/Ecuador?Utm_Content=Bcp_As_Educational_E cuador_Nationalmedia_Awareness\&Utm_Source=Google\&Utm_Medium=Cpc\&Utm_C ampaign=Ecuador_-_Brand-Bcp_All-S\&Utm_Term=Caterpiller. Obtenido De Http://Www.Conexionescat.Com/Inicio/Ecuador?Utm_Content=Bcp_As_Educational_E cuador_Nationalmedia_Awareness\&Utm_Source=Google\&Utm_Medium=Cpc\&Utm_C ampaign=Ecuador_-_Brand-Bcp_All-S\&Utm_Term=Caterpiller.

Catr. (Septiembre De 2016). Http://Www.Tracsa.Com.Mx/Fichas-Tecnicas/Cat-966h.Pdf.

Cohen, R. (2003). Manual De Entrenamiento Para El Piloto De Automovilismo, Planificación Y Organización Del Entrenamiento. Madrid, España: Librerías Deportivas Esteban Sanz.

Cohen, R. (25 De Agosto De 2016). Manual De Entrenamiento Para El Piloto De Automovilismo. España: Librerías Deportivas Esteban Sanz, S.L.

Diario El Comercio. (11 De Diciembre De 2011). El Comercio. Obtenido De Http://Www.Elcomercio.Com/Deportes/Carburando/Fernando-Madera-HistoriaReferente-Local.Html

El Comercio. (11 De Noviembre De 2011). El Automovilismo En El Ecuador. Obtenido De Http://Www.Elcomercio.Com/Deportes/Carburando/Automovilismo-Ecuador.Html

El Comercio. (16 De Abril De 2013). El Comercio. Obtenido De Http://Www.Elcomercio.Com/Deportes/Daniel-Galarza-Dio-Salto-Al.Html

Harre, D. (1987). Teoría Del Entrenamiento Deportivo. La Habana: Stadium Srl.

Inen. (2002). Gestión Ambiental, Aire, Vehículos Automotores. Quito: Inen.

Johann Moncayo, Sebastián Olmos. (Abril De 2017). Entrevista A Pilotos Y Copilotos. Ingenieros Graduados De La Uide. Guayaquil, Guayas, Ecuador: Universidad Internacional Del Ecuador, Sede Guayaquil. Facultad De Ingeniería Automotriz.

Martínez, V. (2016). Maestría En Educación Física. Pruebas De Salud. Guayaquil, Guayas, Ecuador: Universidad Autónoma De Madrid.

Martínez, V. (Octubre De 2016). Pruebas De Condición Física Para La Salud Usm (Bafs). Guayaquil, Guayas, Ecuador: Universidad Autónoma De Madrid.

Nicholas, H. (2010). Manual De Reparación Y Mantenimiento Para Maquinaría Pesada. Graw Hill. 
Organización Mundial De La Salud. (Octubre De 2006). Constitución De La Organización $\begin{array}{lllll}\text { Mundial De } & \text { La } & \text { Salud. } & \text { Obtenido }\end{array}$ Http://Www.Who.Int/Governance/Eb/Who_Constitution_Sp.Pdf

Revista Motor. (S.F.). Nuevas Tendencias Automotrices De Los Últimos Tiempos. Revista Motor. Roberto Hernández Sampieri, Carlos Fernández Collado \& Pilar Baptista Lucio. (2006).

Metodología De La Investigación. Obtenido De Https://Competenciashg.Files.Wordpress.Com/2012/10/Sampieri-Et-Al-Metodologia-DeLa-Investigacion-4ta-Edicion-Sampieri-2006_Ocr.Pdf

The International Council on Clean Transportation. (2011). Introducción A La Refinación Del Petróleo Y Producción De Gasolina Y Diésel Con Contenido Ultra Bajo De Azufre. Theicct, 1-45.

Tracsa. (Agosto De 2017). Http://Www.Tracsa.Com.Mx/Fichas-Tecnicas/Cat-966h.Pdf. Obtenido De Http://Www.Tracsa.Com.Mx/Fichas-Tecnicas/Cat-966h.Pdf.

Vanegas, E. (01 De Marzo De 2016). Informe Proyecto Ecopaís-01-03-16-1. Obtenido De Lineamientos Principales Del Proyecto Eco país: Https://Es.Scribd.Com/Document/357908420/Informe-Proyecto-Ecopais-01-03-16-1

Yépez, V. (2012). Coste, Producción Y Mantenimiento De Maquinaría Para La Construcción. Valencia. 